\title{
Pneumomediastinum from nasal insufflation of cocaine
}

\author{
Brian T. Kloss • Claire E. Broton • Elliot Rodriguez
}

Received: 15 March 2010 /Accepted: 13 June 2010 /Published online: 21 August 2010

(C) Springer-Verlag London Ltd 2010

\begin{abstract}
Chest pain is a common presenting symptom of cocaine users to the emergency department that requires a thorough work up. Pneumomediastinum is an uncommon complication of cocaine abuse that occurs more commonly when cocaine is smoked, but can also occur when cocaine is nasally insufflated. Our case report presents a patient with pneumomediastinum secondary to cocaine insufflation and reviews the necessary diagnostic tests that must be performed to rule out secondary pneumomediastinum, a severe life-threatening condition. Our case is unique, as it is one of a few reported cases of pneumomediastinum occurring after the use of intranasal cocaine.
\end{abstract}

Keywords Pneumomediastinum - Nasal insufflation . Cocaine

\section{Case}

A previously healthy 22-year-old male presented to the ED with a chief complaint of chest pain after having been on a cocaine and alcohol binge for the past $12 \mathrm{~h}$. He had recently used approximately $7 \mathrm{~g}$ of intranasal cocaine and drank a pint of gin. The pain awoke him from sleep and was

B. T. Kloss $(\bowtie) \cdot$ C. E. Broton $\cdot$ E. Rodriguez

Department of Emergency Medicine, Upstate Medical University, 550 East Genesee Street,

Syracuse, NY 13202, USA

e-mail: klossb@upstate.edu

C. E. Broton

e-mail: brotonc@upstate.edu

E. Rodriguez

e-mail: rodrigue@upstate.edu described as: constant, substernal, pressure-like, 10/10 in severity, and exacerbated by movement and deep inspiration. The pain was alleviated with rest and IV opiates.

Vital signs were stable and as follows: HR: 68, BP: 124/ 79, Sp02 99\% on $21 \mathrm{NC}$ and RR: 16. On physical exam the patient was $\mathrm{A} \& \mathrm{Ox} 3$, normocephalic with some rhinorrhea, PERRLA and had normal S1S2 without murmurs; the lungs were clear and the abdomen was soft and non-tender. Pulses were equal and symmetrical bilaterally, and the skin warm and dry. CBC and BMP were unremarkable, with CIPs negative $\mathrm{x}$ 1, and the EKG showed a normal sinus rhythm without arrhythmia or ST segment changes. The AP chest $\mathrm{x}$-ray revealed a pneumomediastinum, which was confirmed by a CT of the thorax. A barium swallow was also performed to rule out esophageal involvement, and given the heavy cocaine use leading up to his condition, a CT reconstruction of the nasopharynx and respiratory system was obtained to rule out perforation (Figs. 1, 2, 3, 4).

\section{Discussion}

Chest pain is a common presenting symptom of cocaine users in the emergency department; however, the majority of these cases are not acute myocardial infarctions, and other potential causes must be considered [1]. Pneumomediastinum, free air in the mediastinum, is an uncommon, but well-established complication of cocaine abuse. A number of cases of pneumomediastinum following cocaine smoking have been reported [1-7]; however, there are few reported cases of pneumomediastinum following nasal insufflation of cocaine [8], which was the case in our patient.

Spontaneous pneumomediastinum (SPM) refers to air in the mediastinum in the absence of any obvious source, such 


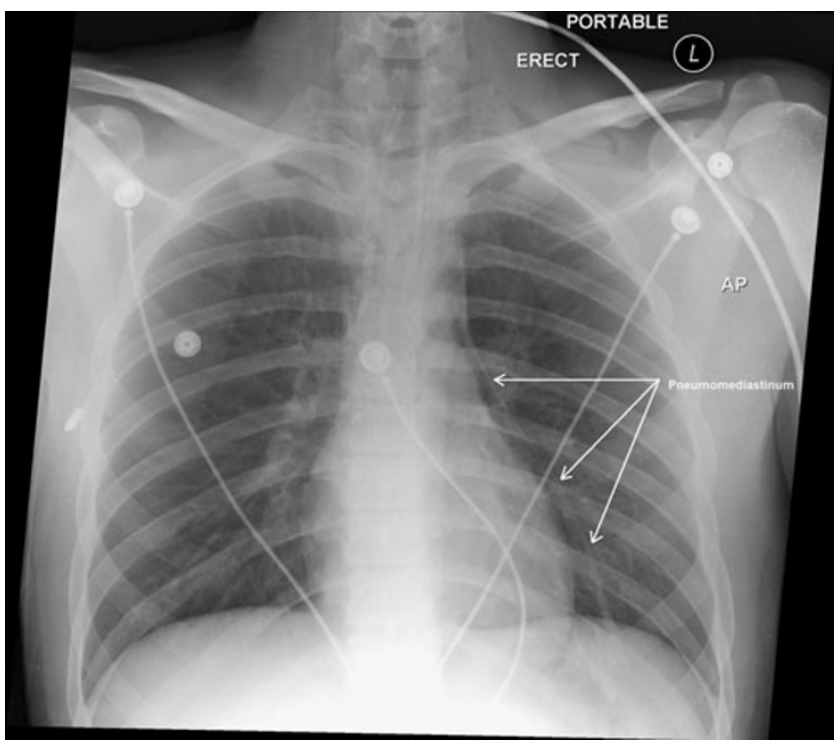

Fig. 1 AP chest X-ray showing pneumomediastinum
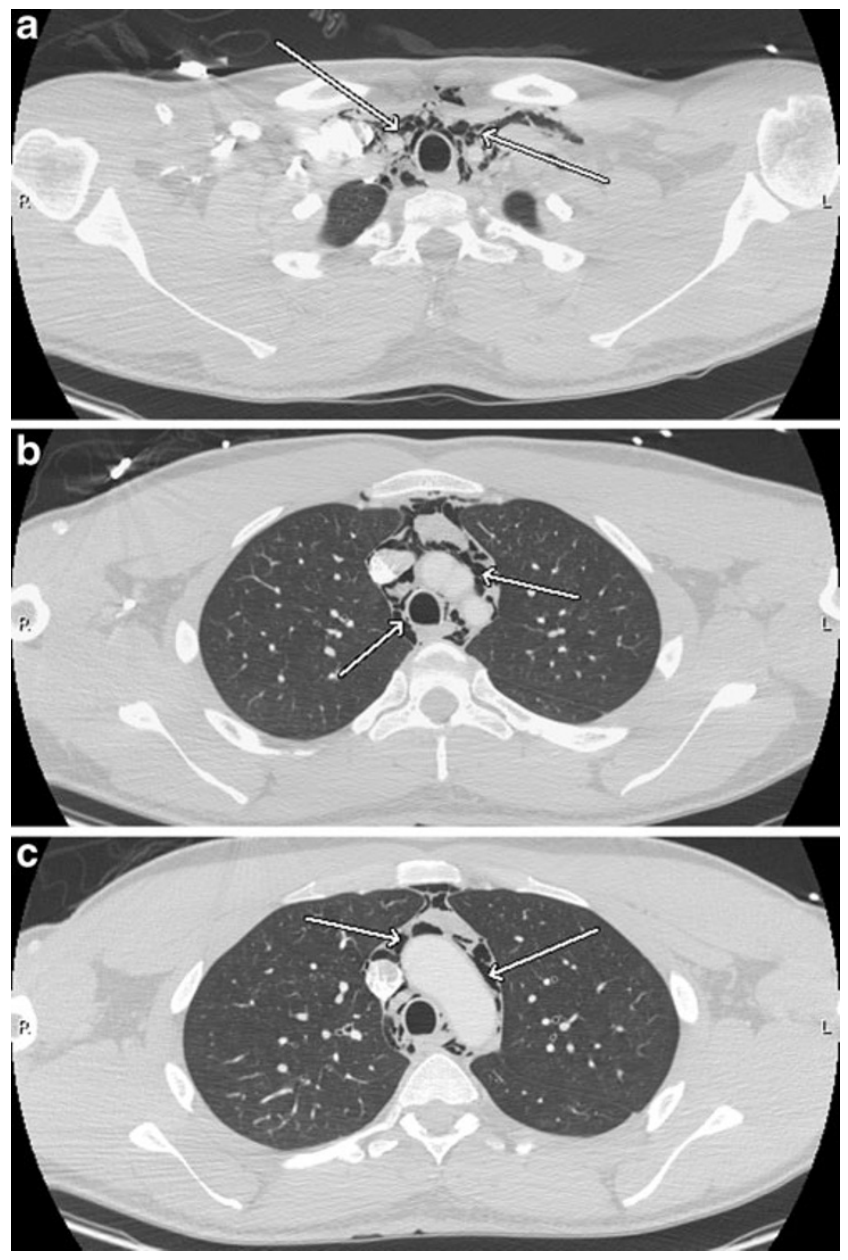

Fig. 2 Cross-sectional CT with arrows showing pneumomediastinum $(\mathbf{a}, \mathbf{b}, \mathbf{c})$

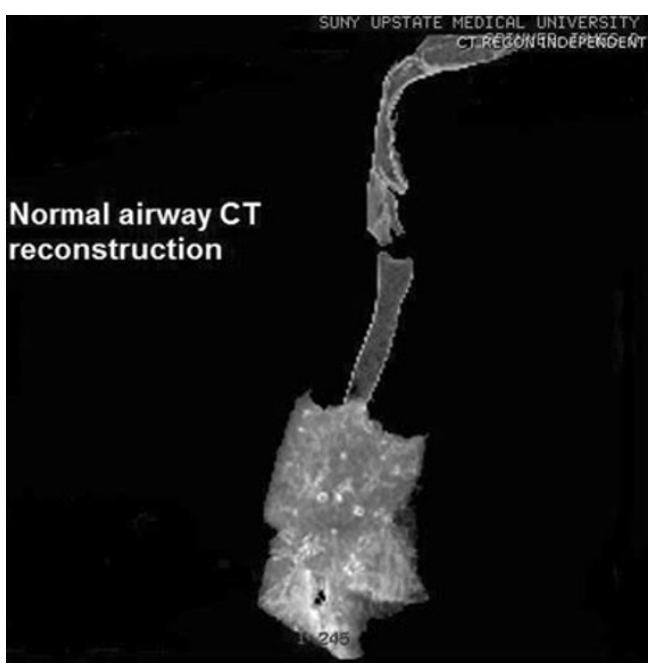

Fig. 3 Normal 3D CT airway reconstruction

as penetrating trauma or severe infection of the respiratory tract. In one retrospective study of admissions of patients with a diagnosis of SPM, the most common precipitating factor was occasional cocaine use, including use within hours of admission [9]. SPM is generally believed to present in young adults after sudden changes in intrathoracic pressure, such as would occur during a Valsalva maneuver. In cocaine users, it is believed that an increase in pressure occurs after smoking, either due to forceful coughing or intentional production of a Valsalva maneuver to increase the absorption and maximize the effect of the drug. When alveoli become over-distended against a closed glottis, the alveoli may rupture into the interstitium and eventually into the mediastinum, producing pneumomediastinum $[10,11]$. The forceful "snorting" and intentional

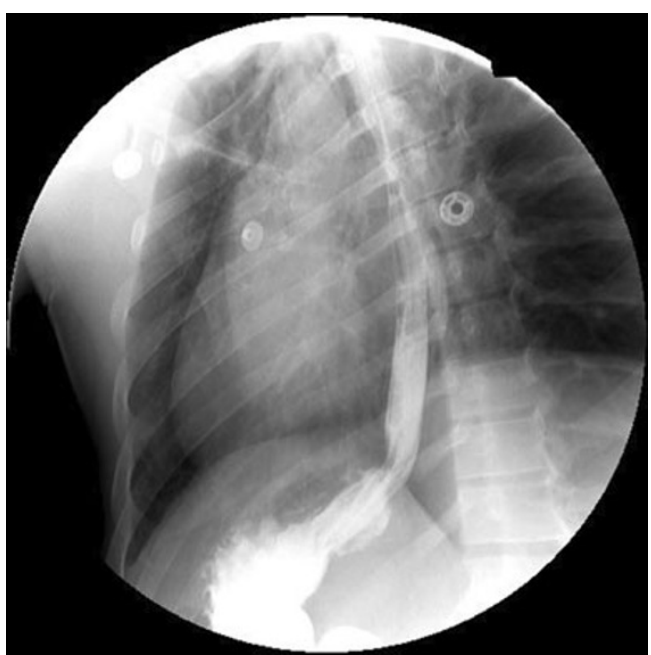

Fig. 4 Normal barium swallow esophagram 
Valsalva maneuver produced following nasal insufflation of cocaine is believed to cause pneumomediastinum by a similar mechanism [8]. It has also been suggested that the direct toxicity of the cocaine on the lung tissue induces injury such as alveolar damage and hemorrhage, which would predispose patients to alveoli rupture and subsequent development of a pneumomediastinum [10].

Young males are the group most commonly affected with SPMs. The most common presenting symptoms of SPM from all causes are: chest pain (54\%), dyspnea (39\%) and cough (32\%) [12]. Additionally, a number of case studies describe patients presenting with an acute sore throat $[3,5,6]$. Common signs of SPM include subcutaneous emphysema of the neck and chest and Hamman's sign, a crackling sound heard on auscultation that correlates with the heart beat [13]. Pneumomediastinum can usually be diagnosed by chest X-ray alone, but CT may be needed in some cases.

It is important to distinguish SPM from a secondary pneumomediastinum with penetration of some part of the airway or esophagus. In a study of 74 patients with pneumomediastinum, the mortality for patients with SPM was $0 \%$, whereas the mortality for patients with secondary pneumomediastinum was 39\% [13]. Secondary pneumomediastinum is associated with greater complications, such as associated pleural effusions and pneumothorax [13]. The respiratory tract and esophagus should be studied radiologically to rule out penetration or leak. Once a patient is identified as having a SPM, the course is generally benign, and management consists mostly of bedrest, pain control and careful follow-up, as was the case with our patient.

\section{Conclusion}

Chest pain is a common presenting symptom of cocaine users to the emergency department that requires a thorough workup. Pneumomediastinum is an uncommon complication of cocaine abuse that occurs more commonly when cocaine is smoked, but can also occur when cocaine is nasally insufflated. When evaluating a patient with chest pain and a history of cocaine use, the physician should be aware of this clinical entity and consider it in their differential diagnosis.

Acknowledgments The authors of this case report did not receive any financial support and have no conflicts of interest to report. Research was performed at the SUNY Upstate Medical Center Department of Emergency Medicine.

Conflicts of interest None.

\section{References}

1. Morris JB, Shuck JM (1985) Pneumomediastinum in a young male cocaine user. Ann Emerg Med 14(2):194-196

2. Cabanas JG, Brice JH, Woodward K (2009) An unrecognized presentation of cocaine-associated pneumomediastinum in the prehospital setting. Prehosp Emerg Care 13(3):384-387

3. Janes SM, Ind PW, Jackson J (2004) Images in thorax: crack inhalation induced pneumomediastinum. Thorax 59:360

4. Maeder M, Ullmer E (2003) Pneumomediastinum and bilateral pneumothorax as a complication of cocaine smoking. Respiration 70:407

5. Sullivan TP, Pierson DJ (1997) Pneumomediastinum after freebase cocaine use. AJR Am J Roentgenol 168(1):84

6. Goldberg REA, Lipuma JP, Cohen AM (1987) Pneumomediastinum associated with cocaine abuse: a case report and review of the literature. J Thorac Imaging 2(3):88-89

7. Uva JL (1997) Spontaneous pneumothoraces, pneumomediastinum and pneumoperitoneum: consequences of smoking crack cocaine. Pediatr Emerg Care 13(1):24-26

8. Luque MA, Cavallaro DL, Torres M, Emmanual P, Hillman JV (1987) Pneumomediastinum, pneumothorax and subcutaneous emphysema after alternate cocaine inhalation and marijuana smoking. Pediatr Emerg Care 3(2):107-109

9. Perna V, Vila E, Guelbenzu JJ, Amat I. Pneumomediastinum: is it really a benign entity? When it can be considered as spontaneous? Our experience in 47 adult patients. Eur J Cardiothorac Surg. 2009; (Epub ahead of print)

10. Gotway MB, Marder SR, Hanks DK, Leung JW, Dawn SK, Gean AD, Reddy GP, Araoz PA, Webb WR (2002) Thoracic complications of illicit drug use: An organ system approach. Radiographics 22 Spec No:S119-S135

11. McCarroll KA, Roszler MH (1991) Lung disorders due to drug abuse. J Thorac Imaging 6(1):30-35

12. Caceres M, Ali SZ, Braud R, Weiman D, Garrett HE (2008) Spontaneous pneumomediastinum: a comparative study and review of the literature. Ann Thorac Surg 86(3):962-966

13. Okereke UN, Weber BE, Israel RH (1999) Spontaneous pneumomediastinum in an 18-year-old black Sudanese high school student. J Natl Med Assoc 91(6):357-359 